\title{
Spectra of equatorial total electron content derived from GPS signals
}

\author{
M. M. J. L. van de Kamp ${ }^{1}$ and P. S. Cannon ${ }^{1,2}$ \\ ${ }^{1}$ Department of Electronic and Electrical Engineering, University of Bath, Claverton Down, Bath, BA2 7AY, UK \\ ${ }^{2}$ Radio Operating Environments, QinetiQ, Malvern, WR14 3PS, UK
}

Received: 25 September 2008 - Revised: 19 March 2009 - Accepted: 9 April 2009 - Published: 15 May 2009

\begin{abstract}
High cadence GPS TEC signals collected on Ascension Island, South Atlantic Ocean, during sunspot minimum, and in Vanimo, Papua New Guinea, over half a solar cycle, have been analysed for their spectral properties. A new parameter $T_{k}$ has been introduced to quantify the strength of TEC irregularities at a scale size of $1 \mathrm{~km}$. The scintillation strength and the spectral index have been analysed as functions of each other, and of local time, season, location and sunspot number. The scintillation strength is highest in autumn and spring in both locations, although the quietest period is summer in Ascension and winter in Vanimo. The scintillation strength decreases with decreasing sunspot number, but is consistently lower in Vanimo than in Ascension. The spectral index decreases with scintillation strength, and increases during the hours of the evening. A method is developed to estimate $T_{k}$ from the large data base of $S_{4}$ measurements.
\end{abstract}

Keywords. Ionosphere (Equatorial ionosphere; Ionospheric irregularities) - Radio science (Ionospheric propagation)

\section{Introduction}

Large electron density gradients, together with winds, electrodynamic forces and instability mechanisms can give rise to regions of strong turbulence, and hence inhomogeneities in the equatorial ionospheric plasma (e.g. Fejer et al., 1999). Such conditions occur most often in the post-sunset period between 18:00 and 24:00 LT (e.g. Aarons, 1982). The inhomogeneities result in refractive index perturbations, which in turn cause phase perturbations on radio waves traversing the region. It is convenient, both conceptually and computationally, to envisage that these perturbations all lie in a thin

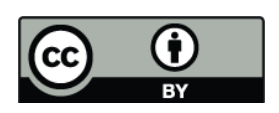

Correspondence to:

M. M. J. L. van de Kamp

(eexmvdk@bath.ac.uk) phase screen located within the ionosphere (e.g. Booker et al., 1950; Rino, 1979a, b). VHF and UHF radio signals from a satellite, which pass through the screen, suffer varying degrees of phase change (but no amplitude change) and a complex diffraction pattern is formed on the ground. As the phase screen or satellite moves, the phase, amplitude and direction of arrival of the received signal at a particular location are seen to vary - this is radio scintillation.

It has been demonstrated that the spectra of electron density irregularities follow a power law between an inner and an outer scale size, and theoretical studies have shown that the associated amplitude and phase scintillation spectra should also follow a power law (e.g. Rumsey, 1975; Rino, 1979a, b). This has been experimentally confirmed on numerous occasions.

According to the above theory, the spectral index $p$ (the magnitude of the spectral slope) of the phase spectrum will be related to the one-dimensional in-situ irregularity spectral index $p_{1}$, according to:

$p=p_{1}+1$

Overlaying this relatively simple description are, however, many second-order effects. For example, a number of studies of irregularity spectra, e.g. by Rino et al. (1981) and Basu et al. (1983), have revealed a pronounced break in the irregularity power law. The latter authors undertook a detailed coordinated study using both topside in-situ satellite data and VHF and GHz ground-based scintillation data. They showed that this breakpoint occurs in both the in-situ and phase spectra at spatial sizes of 500-1000 m, with a long-scale in-situ spectral index $p_{1}$ between 1 and 1.5 (i.e. a phase spectral index $p$ between 2 and 2.5) and a short-scale $p_{1}$ between 3 and 3.5 (i.e. $p$ between 4 and 4.5).

The spectral index has also been variously reported to be dependent on irregularity intensity. For example, Livingston et al. (1981) looked at in-situ and scintillation data representing the long-scale situation and found a systematic decrease

Published by Copernicus Publications on behalf of the European Geosciences Union. 
in spectral index with increasing turbulence. We return to this and related topics later in the paper.

In this paper we describe analyses of Global Positioning System (GPS) signals which have passed through the equatorial crest region. For clarity we point out that we are not analysing the phase spectra as many other authors have done (e.g. Aquino et al., 2007). The original purpose of the analyses was to assess the effect of ionospheric turbulence on the performance of space-based synthetic-aperture radars (van de Kamp et al., 2009). In that context it was appropriate to use, not the scintillation phase or amplitude spectra but the total electron content (TEC) spectra. TEC spectra have the advantage that they can be more readily used to estimate the phase perturbation magnitude and spectra for a synthetic aperture, over a wide frequency range. The disadvantage is that the measurements do not permit an examination of the spectral characteristics at scales significantly less than the size of the Fresnel zone appropriate to the frequency and geometry, which is in the order of $300 \mathrm{~m}$.

We estimate the strength of the TEC irregularities at a scale size of $1 \mathrm{~km}$, the related spectral index, and how these parameters vary with location, sunspot cycle etc. In so doing we will make mention of the assumptions made and the practical problems of the data analyses.

\section{TEC measurement and processing procedure}

The data used for this study were collected on Ascension Island, in the South Atlantic, during sunspot minimum, and in Vanimo, Papua New Guinea, over half a solar cycle. Both locations lie in the equatorial crest region.

\subsection{Measurements on Ascension}

TEC and scintillation data have been collected since August 2004 using a dual-frequency GPS-based Ionospheric Scintillation \& TEC Monitor (Van Dierendonk et al., 1993). It is based on a NovAtel OEM4 GPS module which has been enhanced to provide TEC and scintillation information. Amongst other outputs, this receiver provides TEC estimates at a sampling rate of $1 \mathrm{~Hz}$, which facilitates the spectral estimates. The configuration and deployment of the hardware is described by Fraser (2004) and Fraser et al. (2005). The receiver is located in Georgetown, Ascension Island (latitude $-7.9295^{\circ}$ longitude $-14.4130^{\circ}$; corrected geomagnetic latitude: $-15.15 \mathrm{deg}$ ). Van de Kamp et al. (2009) have described in detail the analysis approach, which will be summarised here.

The TEC, indicating the integrated electron content in a column of unit cross-section, is found from the phase difference of the dual-frequency GPS beacon signal:

$\mathrm{TEC}=\frac{f_{1} / r_{e} c}{f_{1}^{2} / f_{2}^{2}-1} \varphi_{d}$ [electrons $\left./ \mathrm{m}^{2}\right]$ with $f_{1}=$ frequency of $L_{1}$ signal $=1575.42 \mathrm{MHz}, f_{2}=$ frequency of $L_{2}$ signal $=1227.6 \mathrm{MHz}, \quad c=$ velocity of light in vacuum $=299792458 \mathrm{~m} / \mathrm{s}, \quad r_{e}=$ classical radius of an electron $=2.817940325 \times 10^{-15} \mathrm{~m}, \varphi_{d}=$ phase difference between the two signals at different frequencies, defined as:

$\varphi_{d}=\frac{f_{1}}{f_{2}} \varphi_{r 2}-\varphi_{r 1}$

with $\varphi_{r 1, r 2}=$ phases measured at signals $L_{1,2}$.

The inevitable unknown offset in Eq. (2), due to the absence of an absolute phase reference, is estimated with help of the TEC derived from the pseudorange, by ensuring that the average of the carrier-derived TEC over a whole satellite pass equals that of the pseudorange-derived TEC.

The slant TEC is converted to vertical TEC by multiplying by $\sin \varepsilon_{I}$, where $\varepsilon_{I}$ is the elevation angle of the satellite seen from the ionosphere, assumed to be at $350 \mathrm{~km}$ height.

The spectrum of the TEC irregularities is analysed by performing a Fast Fourier Transform on the data over data sections of $17 \mathrm{~min}(1024 \mathrm{~s})$. In order to determine the strength of the scintillation spectrum at a scale size of $1 \mathrm{~km}$, the spectrum is written in wave numbers $g=f / v_{\text {rel }}$, where $f$ is the temporal frequency. $v_{\text {rel }}$ is the relative velocity of the ionospheric pierce point (IPP), defined as the vectorial difference between the velocity of the IPP and the ionospheric drift speed. The ionospheric drift is modelled as varying sinusoidally, (magnetic) eastward during nighttime and westward during daytime, with maxima of $100 \mathrm{~m} / \mathrm{s}$ at local midnight and noon. The vertical ionospheric drift is ignored; this component is strongest around sunset and sunrise, during which time scintillation is weak.

A typical spatial power spectral density $\operatorname{PSD}(g)$ is shown in Fig. 1, as a function of $g$, and of the scale size $L=1 / g$ on the upper axis. TEC is expressed in its standard unit TECU, which is equal to $10^{16}$ electrons $/ \mathrm{m}^{2}$. The power spectral density is, therefore, expressed in $\mathrm{TECU}^{2} / \mathrm{m}^{-1}=\mathrm{TECU}^{2} \mathrm{~m}$.

In the various spectra measured in the presence of irregularities, the highest frequency part was always found to exhibit a steadily decreasing slope. Therefore, a single-slope power-law model, with coefficients $T_{k}$ and $p$, was fitted to the spectrum above $8 \times 10^{-4} \mathrm{~m}^{-1}$ :

$\operatorname{PSD}(g)=T_{k}\left(g L_{k}\right)^{-p}$

where $L_{k}=1000 \mathrm{~m}$. This choice of $L_{k}$ means that $T_{k}$ is a measure of the strength of TEC irregularities at the scale size of $1 \mathrm{~km} . T_{k}$ is closely related (although different) to the phase spectral strength parameter $T$ presented by Secan (1997), which is in turn related to the height-integrated irregularitystrength parameter $C_{k} L$ in WBMOD (Secan, 1996). The parameter $p$ is referred to as the spectral index.

Because the TEC is determined from phase measurements, the measured TEC spectrum may include the effects of phase scintillations due to diffraction by turbulent eddies (Bhattacharyya et al., 2000). This effect will increase for scale 


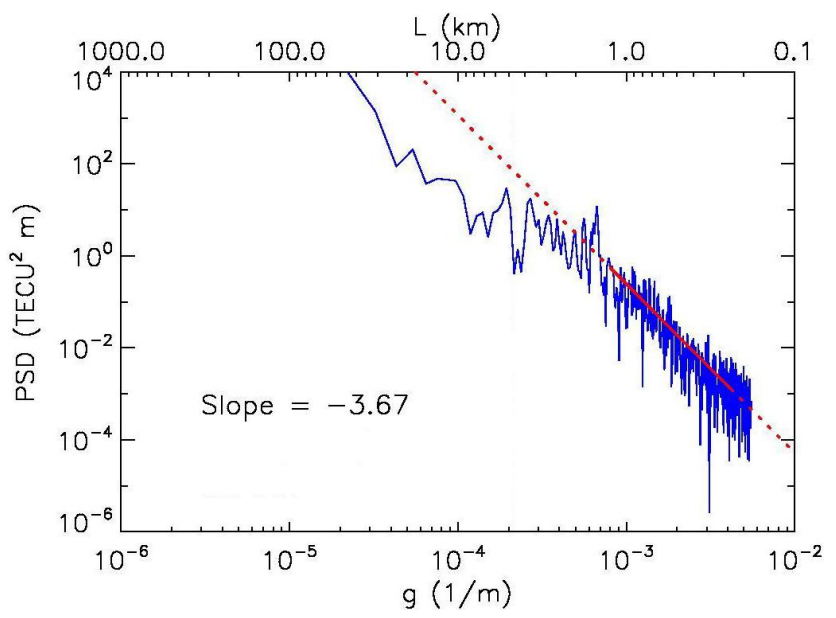

Fig. 1. Spatial spectrum of TEC irregularities, line fitted to the steep high-frequency part, and slope of the fitted line. This spectrum was measured at 22:45 LT, on 26 October 2004.

sizes downward from the first Fresnel zone diameter $L_{F}$. (The first Fresnel zone is the area between the transmit and receive antennas which contributes to coherent signal reception.) Thus, the measured TEC spectra are to some extent affected by scintillation, for spatial frequencies above the inverse of the larger Fresnel zone size (i.e. of the lower frequency) in the ionosphere:

$L_{F 2}=\sqrt{2 c z / f_{2}}$

with $z=$ the distance of the ionosphere from the receiver (which depends on the IPP location). $L_{F 2}$ is typically around $300 \mathrm{~m}$. As can be seen from Fig. 1, the part to the right of this value is only a small part of the measured spectrum. Because of this, the power-law model of Eq. (4) has been fitted to the part of the spectrum between $1 /(1.25 \mathrm{~km})$ and $1 / L_{F 2}$; this model is marked as the solid red line in Fig. 1. The slope of this fitted line, equal to $-p$, is also marked in the graph.

It is worth pointing out that Eq. (4) ignores the two-slope power spectrum described in Sect. 1, but given that Basu (1983) did not measure a steeper gradient until the scale size fell below $500 \mathrm{~m}$, we would not expect to see any signature of a two-slope spectrum in our analysis.

Later in this paper, the analysis will make use of the scintillation model published by Rino (1979a). Consequently, two more parameters are needed: the (scalar) effective velocity $v_{\text {eff }}$ of the IPP, which takes into account the orientation and dimensions of the ionospheric irregularities, and the geometry factor $G$. Using the description by Rino (1982), but simplified for the case where the irregularities are assumed to be aligned along the magnetic field lines, these two parameters have been calculated for each measurement in the data set. They were calculated from the following input parameters: the magnitude and direction of $v_{\text {rel }}$, the elevation and azimuth angles of the satellite from the receiver, the lo-

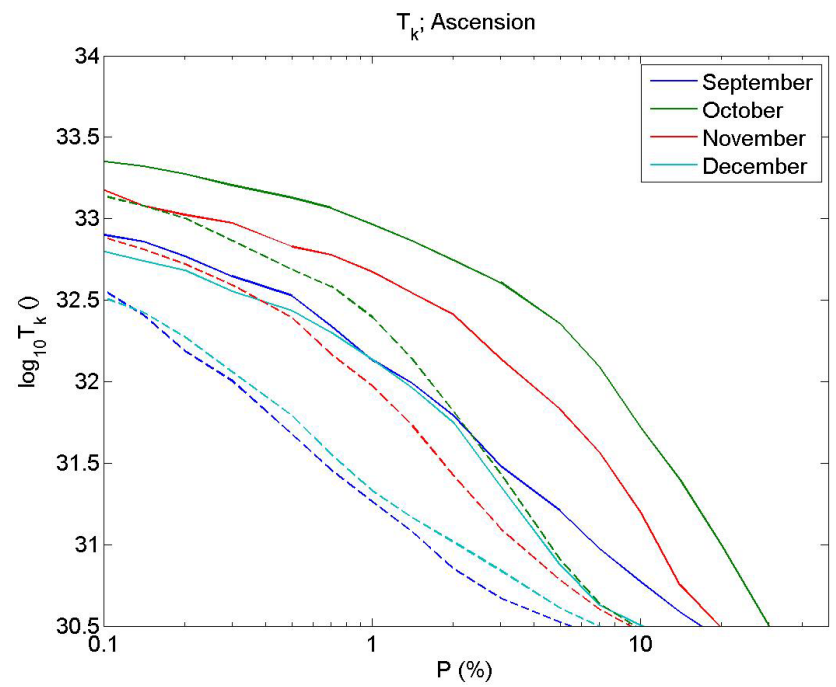

Fig. 2. CDF of $\log _{10} T_{k}$ over Ascension in four months of autumn 2004. Dashed lines: total time; solid lines: only 18:00-24:00.

cal magnetic inclination and declination, the assumed height of the ionosphere, and the axial ratio of the ionospheric irregularities. The latter was set at 30, being the equatorial value used in WBMOD. The resulting $v_{\text {eff }}$ is always between $0 \mathrm{~m} / \mathrm{s}$ and the magnitude of $v_{\text {rel }}$, while $G$ is always larger than 1 , though mostly close 1 .

Using this method, the data from Ascension were analysed over the whole measured period of August 2004January 2009.

\subsection{Measurements in Vanimo}

Similar measurements were also performed by IPS Radio and Space Services, Australia, using a dual-frequency GPS monitor installed in Vanimo, Papua New Guinea (latitude: $-2.6975 \mathrm{deg}$, longitude: $141.3056 \mathrm{deg}$; corrected geomagnetic latitude: $-10.9 \mathrm{deg}$ ). Here, signals were stored at a sampling rate of only $0.5 \mathrm{~Hz}$. The TEC irregularities are analysed in terms of their spatial spectrum, using the same method as for the Ascension data, and again in sections of 1024 s. Using this method, the data from Vanimo were analysed over the whole available period of 2000-2007.

\section{Results}

\subsection{Ascension}

Figure 2 (dashed lines) shows the cumulative distribution of the logarithm of $T_{k}$ over Ascension for the months of September-December 2004. To avoid the effects of terrestrial multipath, all results with elevation angles below 20 degrees have been removed. $T_{k}$ is calculated from TEC expressed in electrons $/ \mathrm{m}^{2}$, i.e. before the conversion 


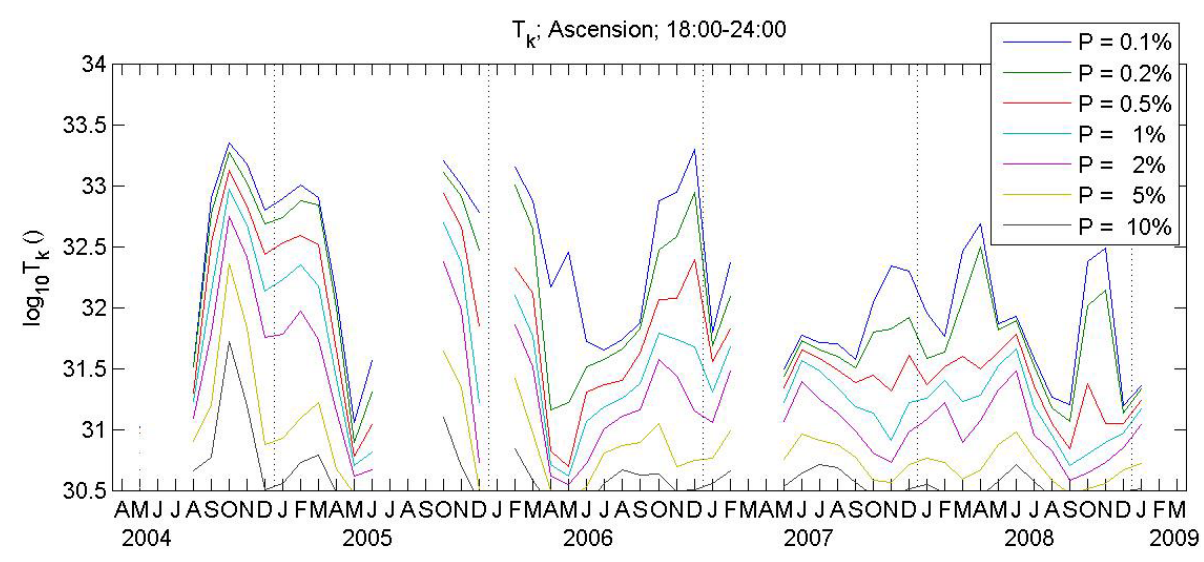

Fig. 3. Values of $T_{k}$ exceeded for various probabilities in Ascension between 18:00 and 24:00, for the months of 2004-2008.

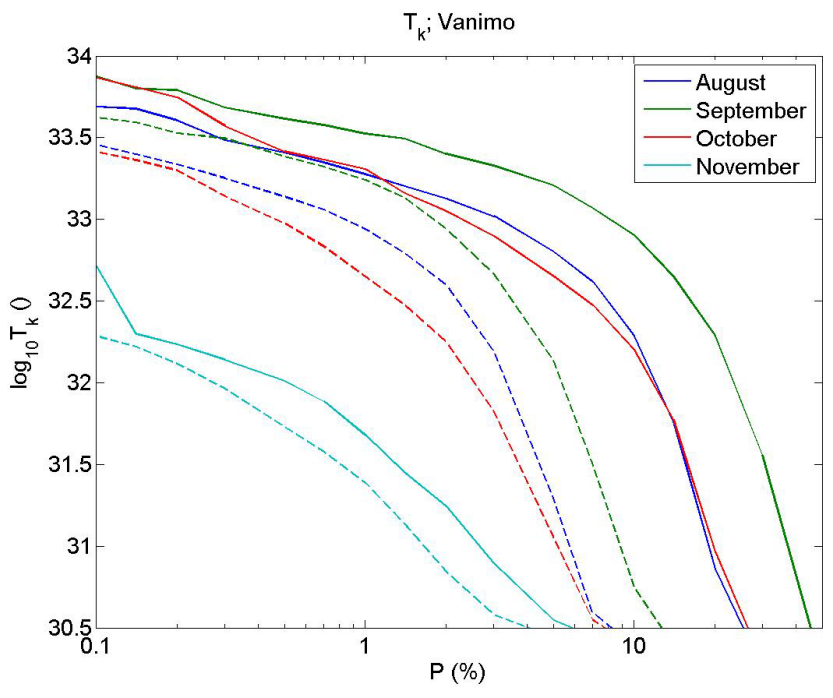

Fig. 4. CDF of $\log _{10} T_{k}$ over Vanimo in four monhs of autumn 2001; full time (dashed lines) and between 18:00 and 24:00 only (solid lines).

to TECU as explained with Fig. 1 ; hence the unit of $T_{k}$ is (electrons $\left./ \mathrm{m}^{2}\right)^{2} \mathrm{~m}$.

It can be seen in Fig. 2 that $T_{k}$ is typically in the order of $10^{31}$, but can reach over $10^{33}$. Scintillation is strongest in the middle of the evening, around 20:00-22:00 LT, while during the day it is very weak, due to the underlying E-region shorting out the polarization fields above. Because of this, Fig. 2 also shows (solid lines) the CDF of $T_{k}$ over just the evening hours (18:00-24:00). As expected, evening probabilities for values for $\log _{10} T_{k}>31.5$ are four times higher than over the full day. $\log _{10} T_{k}$ exceeded 33.0 for $1 \%$ of the time, and 31.7 for $10 \%$ of the time in the evening hours in October 2004.

Figure 3 shows the values of $T_{k}$ exceeded for various probability levels in the evening hours over the months from August 2004 to January 2009, during which time the sunspot number fell from $\approx 50$ to 0 . October 2004 was clearly the most active month. The irregularities started quite suddenly in the beginning of October, after which they decreased progressively until the end of the year. In February and March 2005, a few more active periods were observed, after which the irregularity probability dropped to low values in late spring and summer.

In spite of the gaps in the data, most years show a similar pattern. In all years, autumn and spring were the most active periods, with slight dips in winter and very quiet periods in the summers. This mid-Atlantic seasonal variation is in agreement with Groves et al. (1997) and Nishioka et al. (2008). The equinoctial peaks can be explained considering that the probability of occurrence of plasma bubbles, with the irregularities they cause, is highest when sunset times at either side of the equator are simultaneous (Nishioka et al., 2008).

As expected, the value of $T_{k}$ decreases with decreasing sunspot number. The value of $\log _{10} T_{k}$ exceeded for $1 \%$ of the evening hours decreases, from 33.0 in the autumn of 2004, to around 31.0 in 2008.

\subsection{Vanimo}

TEC variations were analysed for the data from Vanimo over the period from 2000 to 2007 , when the sunspot number decreased from maximum, $\approx 170$, to $\approx 5$. The same elevation angle window was used as for the Ascension data. Figure 4 shows the CDF of $T_{k}$ (dashed lines) for August-November 2001, close to sunspot maximum. For the same exceedence probabilities, $\log _{10} T_{k}$ in September 2001 over Vanimo was roughly $0.5-1.5$ higher than in October 2004 over Ascension.

The solid lines in Fig. 4 show the CDF of $T_{k}$ for the evening hours only. $\log _{10} T_{k}$ exceeded 32.2 for at least $10 \%$ of the evening hours during August to October 2001.

Figure 5 shows the values of $T_{k}$ exceeded for various probability levels in the evening hours over the months from 2000 to 2007. This figure shows that, as in Ascension, spring 


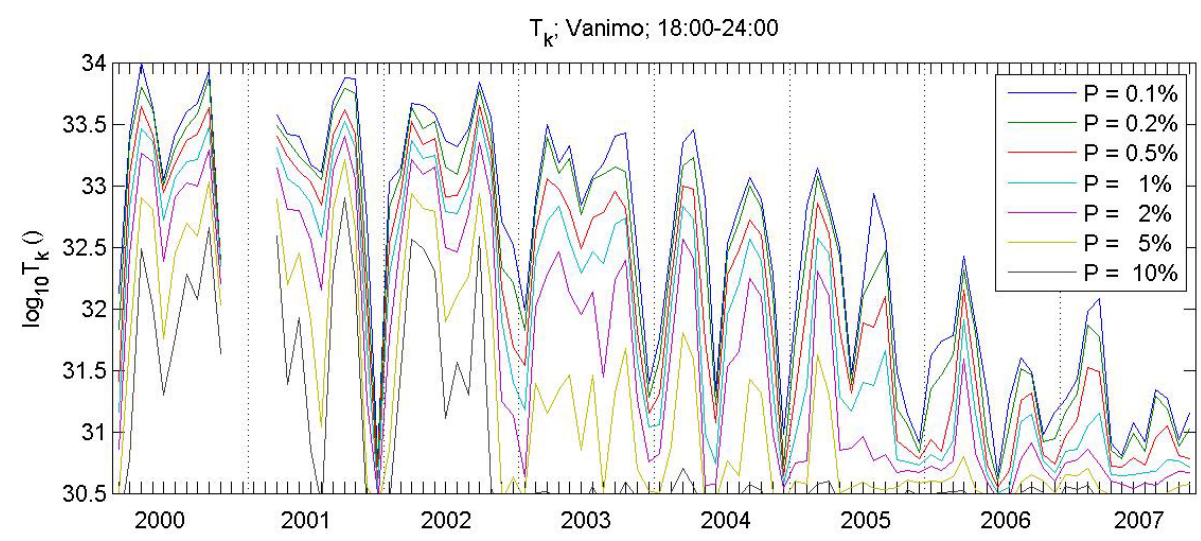

Fig. 5. Values of $T_{k}$ exceeded for various probabilities between 18:00 and 24:00 over Vanimo, for the months of 2000-2007.

and autumn are the most active periods. This is consistent with Fang and Liu (1983) and Cervera et al. (2001), who reported equinoctial peaks in scintillation activity for the Australian/Asian longitude sector.

Comparing this figure with Fig. 3, it can be seen that simultaneous values are generally lower than over Ascension. For instance, in October 2004, $\log _{10} T_{k}$ exceeded for the same probabilities was about 0.6 lower than over Ascension. Also note that over Vanimo, winters were quieter than summers, while over Ascension, summers were the quietest periods.

As over Ascension, irregularities decrease with the decreasing sunspot number. The value of $\log _{10} T_{k}$ exceeded for $1 \%$ of the evening hours decreases from around 33.4 in spring/autumn 2000 to around 30.8 in 2007.

\subsection{The spectral index}

The spectral index $p$ of the TEC spectrum at equatorial latitudes is an interesting one. Nickisch (2004) addressed this for a high-latitude scenario by looking at the slope of GPS and Early Warning Radar data. He determined that the average TEC spectral index was 2.7 , a value close to 3 , which is the value for the high-latitude phase spectral index in the WBMOD scintillation model. He concluded that the scintillation climatology inherent in WBMOD could be extended to quantify the slope in TEC irregularities. Like us, Nickisch (2004) appears not to have seen a two-slope spectrum - perhaps not surprisingly given that the sampling rate restricted the measurements to the longer spatial wavelength regime. In equatorial latitudes, WBMOD version 13 (Secan, 1996) uses a fixed index of 2.5, and GIM (Béniguel, 2002) uses 3 (Y. Béniguel, private communication, 2008); Basu et al. (1983) found values of 3 and 3.5 appropriate.

\subsubsection{Variation with turbulence}

The spectral index of TEC irregularities is the parameter $p$ resulting from the fitting of the power law of Eq. (4) to the measured PSD. Figure 6 shows a scatterplot of $p$, as a func-

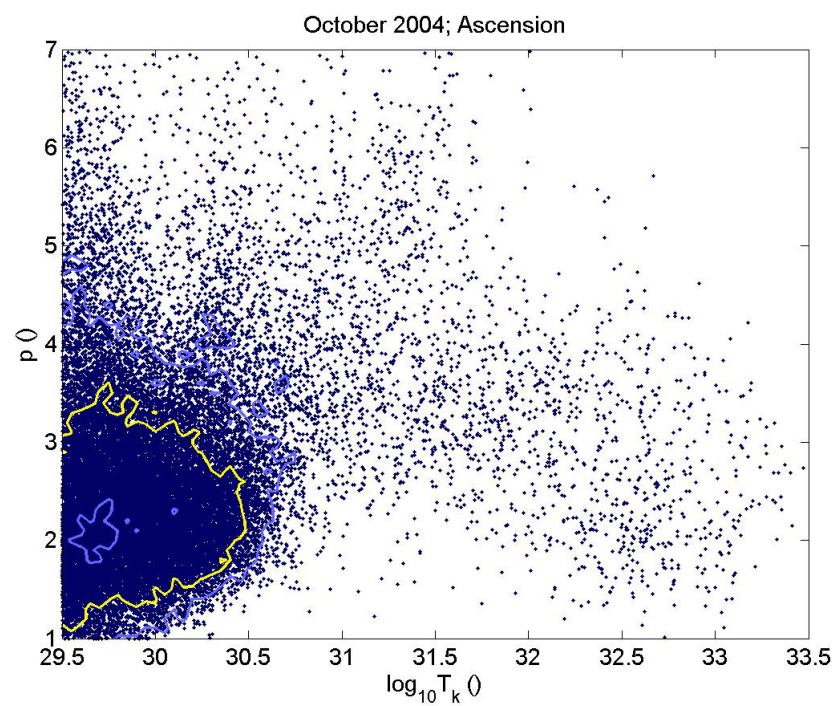

Fig. 6. Scatterplot of $p$ and $T_{k}$ for Ascension.

tion of $T_{k}$ for Ascension Island in October 2004, the month of maximum activity. The overlaid contour lines indicate densities of data points, logarithmically increasing as 10, 30, 100, 300 , etc. points per grid square. Figure 7 shows the same data in a scatterplot of $p$ versus $S_{4}$.

This figure shows that for significant scintillation $\left(T_{k}>10^{31}\right), p$ decreases with increasing $T_{k}$. However, for low scintillation levels, the values are more varied, and the majority of data give a low value of $p$ (around 2), although the highest values $(\sim 5-7)$ also occur for low scintillation. Figure 7 shows a similar result versus $S_{4}$. The same analysis was performed on the Vanimo data, from which a similar dependence of the spectral index $p$ on $T_{k}$ was observed. Wernik et al. (2007), who analysed spectra of in-situ measurements of high-latitude ionospheric turbulence, found a similar increase/decrease of the spectral index with turbulence strength. 


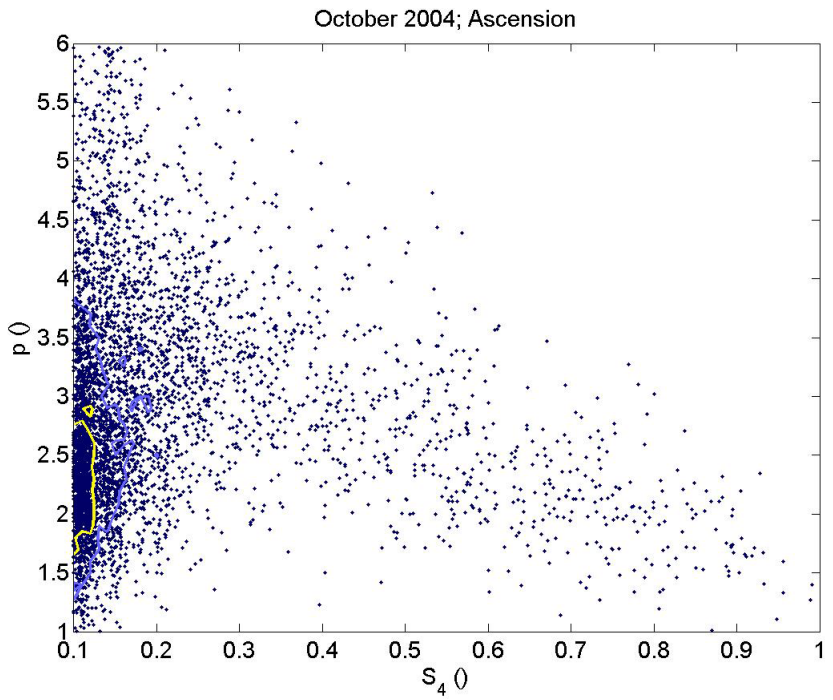

Fig. 7. Scatterplot of $p$ and $S_{4}$ for Ascension.

It should be noted, however, that the points on the lefthand side of Fig. 6 are influenced by thermal receiver noise, phase noise and multipath. The median value for $T_{k}$ in quiet conditions (summer-daytime) is $10^{29.8}$; this provides a good estimate of the noise floor. The noise reduces the measured spectral index for values of $T_{k}$ up to about a decade above the noise floor, i.e. for $T_{k}<10^{31}$.

As for the decrease of the spectral index with increasing turbulence strength, this effect has been widely reported, e.g. by Rino (1979a), Rino et al. (1981), Livingston et al. (1981) and most recently by Wernik et al. (2007). According to Rino (1979a), this effect may be due to the rapid phase changes driving the measured phase spectrum toward that of a discontinuous random process.

In the central region in the range of $S_{4}$ between 0.2 and 0.6 , where the measurements are not unduly influenced by the effects of noise nor by discontinuities, the average $p$ from the data of Fig. 7 is 3.4, which is much larger than the climatological value of 2.5 used in WBMOD (Secan, 1996), larger than the value of 3 used in GIM, and comparable to the value measured by Basu et al. (1983). In the range of $S_{4}$ between 0.2 and 1.0, the average $p$ is 3.2, which is closer to the values for the phase spectral index employed in climatological models.

\subsubsection{Variation with solar cycle, time of day and location}

To ascertain whether the spectral index depends on any other parameter, the median spectral index $p$ has been calculated from the long-term data from both Ascension and Vanimo. In order to avoid the dependence on scintillation strength mentioned earlier, the median $p$ is calculated from only the central region of $T_{k}$, in the range $31.1 \leq \log _{10} T_{k} \leq 31.8$. Figure 8

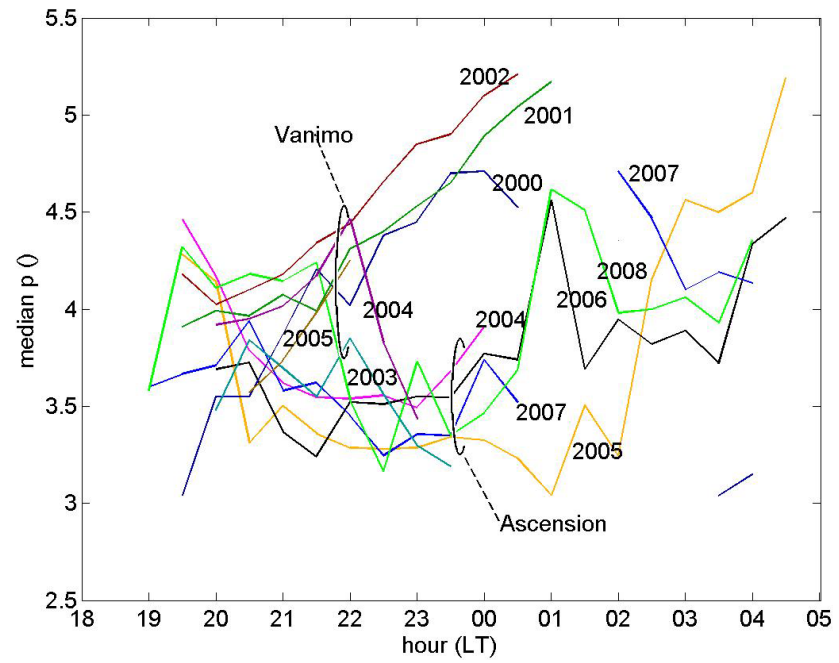

Fig. 8. The median spectral index $p$ in Ascension and Vanimo as functions of the hour of the night.

shows the resulting spectral indices as functions of the hour of the day and the year. In this graph, only the data points are shown for which the number of (17-min) TEC spectra was at least 50 .

This figure shows a significant variation of the spectral index $p$ over the hours of the night. In Vanimo, in the years 2000-2002, $p$ consistently increased during the hours of night, from values of about 3.5-4.0 at 20:00, to above 5.0 at 01:00. In 2003-2005, $p$ showed similar values to the preceding years up to 23:00. However, in these years, the lower ionospheric activity, combined with the relatively low $\mathrm{C} / \mathrm{N}$ ratio of the Vanimo measurements, resulted in available data during a shorter period of the night, and more variable results during this period, so that a steady increase of $p$ with the hour cannot be observed. In 2004-2008 in Ascension, $p$ remained steady at a level of about 3.5 in the early night, similar to the values in Vanimo in the corresponding hours, and increased after about 23:00.

The spectral index was in Ascension consistently 0.5-1.0 lower than in Vanimo during the same hours. Unfortunately however, the shortage of significant coincident data restricts our analysis. The measurements in Vanimo often contained too much noise to study the spectral index near solar minimum, when ionospheric activity was low (2003-2007), while those at Ascension had not yet commenced at solar maximum, when it was high (2000-2003). Thus, there is too little overlap to verify reliably whether the different spectral indices observed between solar minimum in Ascension and solar maximum in Vanimo are due to the different locations or due to the different years/ionospheric activity levels.

The increase of $p$ with the hour of the evening suggests a decrease of small-scale structures compared to the larger ones. This is in agreement with results found by other researchers: 
Franke et al. (1984) analysed several isolated scintillation events measured at Ascension Island at 1.5 and $3.9 \mathrm{GHz}$. They measured the spectral index of the scintillation spectrum and the frequency scaling index of $S_{4}$ between the two frequencies, which parameters according to weak scattering theory are linearly related. They found that both of these parameters were on average larger for later hours, on any evening.

Mullen et al. (1985) analysed ionospheric scintillation, measured at Ascension at 0.26, 1.5 and $3.9 \mathrm{GHz}$. They found that scintillation typically reached its maximum simultaneously at the three frequencies, but decayed first at the highest, and last at the lowest frequency. They conclude from this that the lifetime of short-scale irregularities is several hours shorter than that of the larger scales. Since the appropriate range of Fresnel zone sizes $(175-700 \mathrm{~m})$ mostly overlaps with the range over which the power law is fitted to the TEC measurements in Sect. 2 (about 200-1250 m), these findings agree with the increase of the spectral index with the hour found in this study.

Wernik et al. (2007) analysed spectra from in situmeasurements of ionospheric turbulence at latitudes above $50^{\circ}$. They found that for latitudes between $50^{\circ}$ and $70^{\circ}$, the spectral index increased with local time from about 18:00 to midnight, and decreased after that. In the winter season, another daily maximum of $p$ was observed around local noon.

\section{Deriving $T_{k}$ from $S_{4}$}

In order to compare the results in this paper with data from different measurements, it is useful to find a relation between $T_{k}$ and other scintillation parameters. The parameter $S_{4}$, defined as the 1-min standard deviation of signal level divided by the mean signal level, is a widely used parameter, for which statistics measured at various sites are available, going back many years. It was also recorded in the measurements on Ascension.

In this section a relation between $T_{k}$ and $S_{4}$ is established from the model developed by Rino (1979a) for the spectrum of phase variations due to weak scatter measured on a transionospheric link. The approach is similar to that adopted in an earlier synthetic aperture radar study (van de Kamp et al., 2009).

The model by Rino for the spectrum of phase variations gives:

$\operatorname{PSD}_{\varphi}(f)=\frac{r_{e}^{2} \lambda^{2}}{\left(f_{L}^{2}+f^{2}\right)^{p / 2}} \frac{z_{I}}{\sin \varepsilon_{I}} G C_{s} \frac{\sqrt{\pi} \Gamma\left(\frac{p}{2}\right)}{(2 \pi)^{p+1} \Gamma\left(\frac{p+1}{2}\right)} v_{\mathrm{eff}}^{p-1}(6)$

where $f_{L}=$ frequency corresponding to the outer scale of turbulence, $\lambda=$ wavelength, $C_{S}=$ parameter representing the strength of turbulence, $z_{I}=$ length of the path through the ionosphere.
The model for intensity scintillation (Rino, 1979a) gives:

$S_{4}^{2}=r_{e}^{2} \lambda^{2} \frac{z_{I}}{\sin \varepsilon_{I}} C_{s} Z^{(p-1) / 2} \frac{\Gamma\left(\frac{5-p}{4}\right)}{2 \sqrt{\pi}\left(\frac{p-1}{2}\right) \Gamma\left(\frac{p+1}{4}\right)} J$

$Z=\frac{\lambda}{4 \pi \sin \varepsilon_{I}} \frac{z z_{s}}{\left(z+z_{s}\right)}$

where $z_{s}=$ distance from the irregularity layer to the satellite, $z=$ distance from the irregularity layer to the receiver.

$J$ is a combined geometry and propagation factor, which in the case of an infinite axial ratio, or a two-dimensional irregularity model, becomes (Rino, 1979a):

$J=\frac{\Gamma\left(\frac{p}{2}\right)}{\sqrt{\pi} \Gamma\left(\frac{p+1}{2}\right)}$

The assumption of an infinite axial ratio may be justified in the case of equatorial scintillations, where the irregularities are elongated along the magnetic field lines.

Equation (6) is converted to the spectrum of TEC variations by dividing by $r_{e}^{2} \lambda^{2}$, converted to that of vertical TEC by multiplying by $\sin ^{2} \varepsilon_{I}$, expressed in terms of scale size $g=f / v_{\text {rel }}$, and approximated for scale sizes much smaller than the outer scale of turbulence; the result is:

$\operatorname{PSD}_{\mathrm{TEC}}(g)=\frac{G C_{s} z_{I} \sin \varepsilon_{I}}{g^{p}} \frac{\sqrt{\pi} \Gamma\left(\frac{p}{2}\right)}{(2 \pi)^{p+1} \Gamma\left(\frac{p+1}{2}\right)}\left(\frac{v_{\mathrm{eff}}}{v_{\mathrm{rel}}}\right)^{p-1}$

Combining this with Eqs. (4) and (7) gives $T_{k}$ as a function of $S_{4}$ :

$T_{k}=\frac{S_{4}^{2} G L_{k}^{p}\left(\sin \varepsilon_{I}\right)^{2}}{r_{e}^{2} \lambda^{2} Z^{(p-1) / 2}} \frac{\left(\frac{p-1}{2}\right) \Gamma\left(\frac{p+1}{4}\right) \sqrt{\pi}}{\Gamma\left(\frac{5-p}{4}\right)(2 \pi)^{p}}\left(\frac{v_{\text {eff }}}{v_{\text {rel }}}\right)^{p-1}$

It may be noted that the intensity fluctuations represented by $S_{4}$ are caused by TEC irregularities within the Fresnel zone only, and therefore $S_{4}$ represents only the part of the TEC spectrum for scale sizes smaller than the Fresnel scale size of the GPS beam (which is smaller than $L_{k}$ ). Equation (11) takes this 'scale size scaling' into account, by the factors $L_{k}$ and $Z$. Assuming the different spectral parts are proportional, this will not lead to an underestimate of $T_{k}$ from $\mathrm{S}_{4}$.

Equation (11) has been used to estimate $T_{k}$ from values of $S_{4}$ and $p$ with the values of $\varepsilon_{I}, G, v_{\text {rel }}$ and $v_{\text {eff }}$ calculated from the geometrical configuration. The data analysis has been restricted to that collected during October 2004 on Ascension Island. The $S_{4}$ values are averaged over the same 17min periods for which $T_{k}$ was calculated in Sect. 2.1. The following parameter values were used in Eq. (11): $L_{k}=1000 \mathrm{~m}$, $\lambda=0.19029 \mathrm{~m}$ ( $L_{1}$ signal), $z=350 \times 10^{3} \mathrm{~m}, z_{s}=19.8 \times 10^{6} \mathrm{~m}$ (the average height of a GPS satellite above the ionosphere). The result is compared to $T_{k}$ derived from the Ascension 


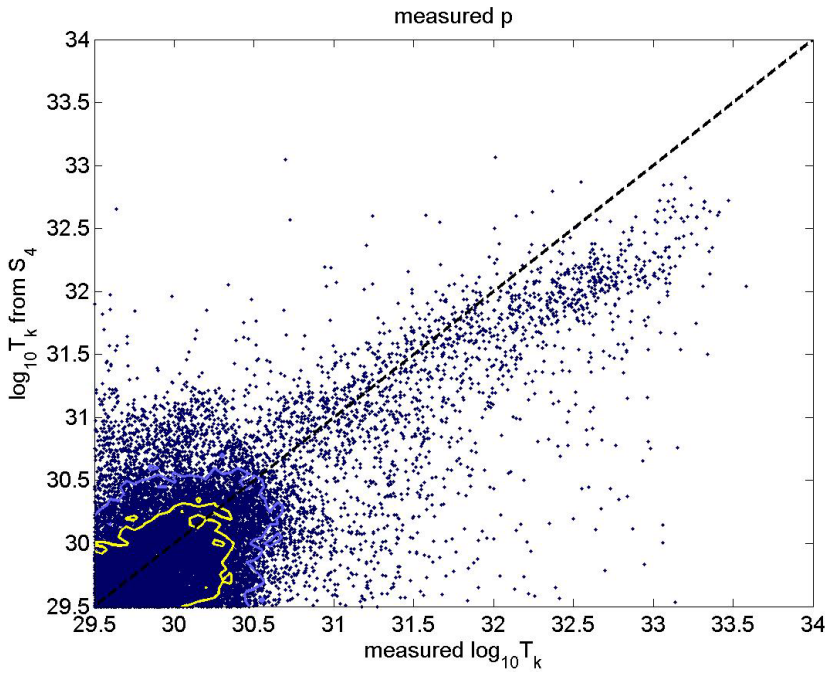

Fig. 9. $\log _{10} T_{k}$ estimated from $S_{4}$, compared to the measured values.

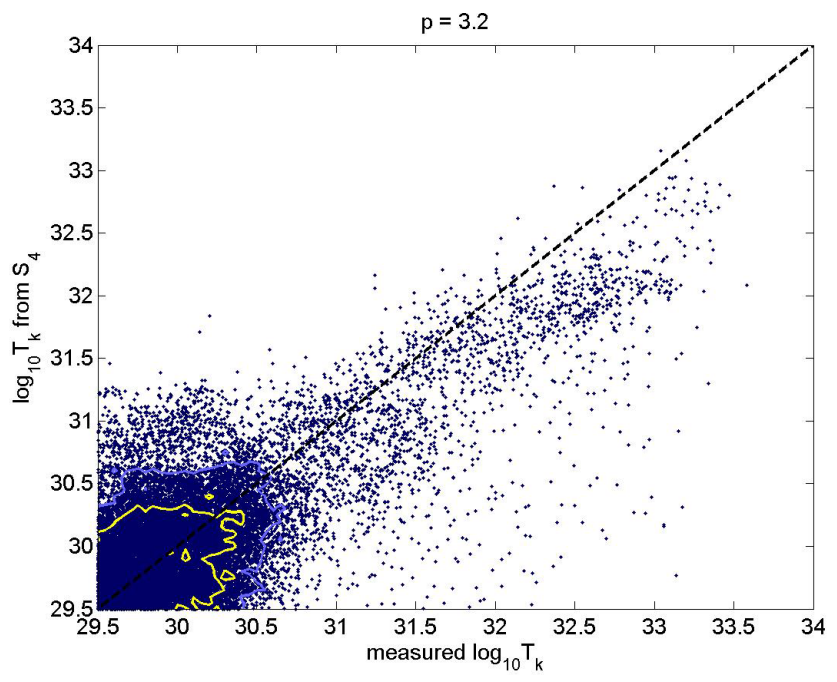

Fig. 10. $\log _{10} T_{k}$ estimated from $S_{4}$ compared to the measured values, using a constant $p=3.2$.

measurements as described in Sect. 2.1, in the scatterplot of Fig. 9. As in Fig. 6, the overlaid contour lines indicate densities of data points in the plot.

Figure 9 shows that Eq. (11), on average, estimates the measured values of $T_{k}$ well for weak scintillation - albeit with a spread of approximately a factor 10 . However, Eq. (11) increasingly underestimates the measured values when $\log _{10} T_{k}$ increases above 31.6 , which corresponds to a value for $S_{4}$ of about 0.25 .

In an applications context, the use of Eq. (11) is most useful to predict $T_{k}$ from $S_{4}$ if no spectral analysis has been carried out to assess $T_{k}$, which means that also the spectral index $p$ is not available. Aquino et al. (2007) identified this issue

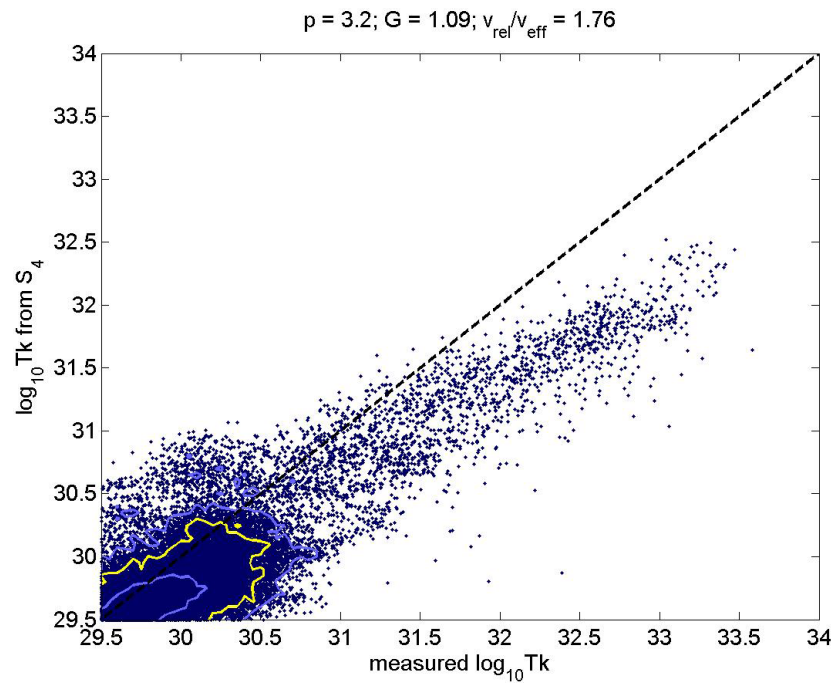

Fig. 11. $\log _{10} T_{k}$ estimated from $S_{4}$ compared to the measured values, using $p=3.2, G=1.09$ and $v_{\text {rel }} / v_{\text {eff }}=1.76$.

in the calculation of the phase parameter $T$ from a database of $S_{4}$. Because of this, Eq. (11) has been re-evaluated using a fixed value of $p$ of 3.2, the average found for $S_{4}>0.2$ in Sect. 3.3.1. The result, shown in Fig. 10, is very similar to Fig. 9: the average error is similar, and the correlation is in fact slightly better. The latter observation indicates that some of the spread in Fig. 9 had been introduced through the failure of the automated process for fitting a spectrum to the data. Given that the method with a fixed $p$ is more practical than the one which led to Fig. 9, this is a useful result.

The estimation of $T_{k}$ from $S_{4}$ was simplified further, by replacing the assessment of $v_{\text {eff }}$ and $G$ from the geometrical setup, which involves quite a complicated calculation (Rino, 1982), by fixed values of $G$ and $v_{\text {rel }} / v_{\text {eff. For this purpose, }}$ the median value of $G$ and $v_{\text {rel }} / v_{\text {eff }}$ over the measurements in Ascension in October 2004 were used, which were $G=1.09$ and $v_{\text {rel }} / v_{\text {eff }}=1.76$.

Using these constant values, Eq. (11) has again been applied; the result is shown in Fig. 11. This figure shows that the underestimation of $T_{k}$ is slightly larger than that in the previous two figures; however, the correlation has improved significantly. It would appear that the scatter of the points in Figs. 9 and 10 was mainly due to uncertainties in the values of $v_{\text {rel }}, v_{\text {eff }}$ and $G$, i.e. uncertainties regarding the geometry of the turbulent ionosphere.

The principal reason for the underestimation of $T_{k}$ from $S_{4}$ for strong scintillation conditions in all three examples, is almost certainly the application of Rino's weak scatter model, which does not accurately represent the stronger range of irregularities. Other contributions likely derive from the adopted parameter values in Eq. (11); these include the assumption made for the geometrical factor $J$ in Eq. (9), and the assumption of a constant spectral index, as well as the 
choice of the fixed values of $G$ and $v_{\text {rel }} / v_{\text {eff }}$ in the case of Fig. 11. Different values for all these parameters (possibly dependent on other parameters), would possibly improve the estimate, however, a more detailed analysis of this would be beyond the scope of this paper.

These results show that, although the method may need to be refined, it is possible to estimate $T_{k}$ from measurements of $S_{4}$, at least for weak scintillation conditions - albeit with low fidelity. This method provides a useful estimate of the spectrum of TEC variations in an equatorial region where only $S_{4}$ measurements have been previously made.

\section{Conclusions}

The results presented in this paper show the statistics of spectral properties of TEC fluctuations during equatorial ionospheric scintillation over two regions, the South Atlantic and Papua New Guinea. Data availability has restricted this analysis to five years near sunspot minimum for the former and half a solar cycle of data for the latter.

The strength of TEC irregularities has been quantified through the introduction of a parameter $T_{k}$, which represents the power spectral density at a scale size of $1 \mathrm{~km}$. It has been found that scintillation strength is as expected strongest in the few hours before midnight. Both over Ascension and Vanimo, scintillation is strongest in the spring and the autumn. Between these periods, Ascension shows very quiet periods in the summer, and Vanimo in winter. Over Ascension, $\log _{10} T_{k}$ exceeded 33.0 for $1 \%$ of the time, and 31.7 for $10 \%$ of the time in the evening hours in October 2004. Over Vanimo, $\log _{10} T_{k}$ exceeded 32.2 for at least $10 \%$ of the evening hours during August to October 2001. Simultaneous exceedence probabilities of $T_{k}$ are lower over Vanimo than over Ascension; for instance, in October $2004 \log _{10} T_{k}$ exceeded for the same probabilities was about 0.6 lower than over Ascension.

As expected, the scintillation occurrence decreases with decreasing sunspot number. Over Ascension, the value of $\log _{10} T_{k}$ exceeded for $1 \%$ of the evening hours decreases, from around 33.0 in the autumn of 2004, to around 31.0 in 2008. Over Vanimo, the same value decreases from around 33.4 in spring/autumn 2000 to around 30.8 in 2007.

The spectral index $p$ decreases with scintillation strength. For weak scintillation the measured spectrum may be flattened by thermal noise. The average spectral index in October 2004 over Ascension for $S_{4}>0.2$ was 3.2. The spectral index increases with the hour of the night. The spectral index is larger over Vanimo close to solar maximum than over Ascension close to solar minimum, but data availability and quality prevents us from determining if this difference is due to year or location.

In recognition of the fact that similar high-resolution TEC data are limited, an analytic approach to calculating the TEC spectral strength parameter from $S_{4}$ has been introduced. It is shown that under weak scattering conditions, $S_{4}$ can be mapped to $T_{k}$ albeit with a spread of a factor 10 . The application of this mapping in a practical application has been explored using average values for the spectral slope and other geometrical parameters. It is shown that these assumptions have a minimal impact on the model's ability to derive $T_{k}$ from $S_{4}$.

Acknowledgements. The authors would like to thank QinetiQ for permitting us to use the data from Ascension Island, and Mike Terkildsen and Phil Wilkinson of IPS Radio \& Space Services, Australia, for the data from Vanimo. The authors would also like to thank A. Bhattacharyya and the referees for their insightful comments.

Topical Editor K. Kauristie thanks two anonymous referees for their help in evaluating this paper.

\section{References}

Aarons, J.: Global morphology of ionospheric scintillation, Proc. IEEE, 70(4), 360-377, 1982.

Aquino, M., Andreotti, M., Dodson, A., and Strangeways, H. J.: On the use of ionospheric scintillation indices as input to receiver tracking models, Adv. Space Res., 40, 426-435, doi:10.1016/j.asr.2007.05.035, 2007.

Basu, S., Basu, S., McClure, J. P., Hanson, W., and Whitney, H.: High Resolution Topside in Situ Data of Electron Densities and VHF/GHz Scintillations in the Equatorial Region, J. Geophys. Res., 88(A1), 403-415, 1983.

Béniguel, Y.: Global Ionospheric Propagation Model (GIM): A propagation model for scintillations of transmitted signals, Radio Sci., 37(3), 4.1-4.10, doi:10.1029/2000RS002393, 2002.

Bhattacharyya, A., Beach, T. L., Basu, S., and Kintner, P. M.: Nighttime equatorial ionosphere: GPS scintillations and differential carrier fluctuations, Radio Sci., 35, 209-224, 2000.

Booker, H. G., Ratcliffe, J. A., and Shinn, D. H.: Diffraction from an irregular screen with application to ionospheric problems, Philosophical Transactions of the Royal Society, Series A, 242, 579-607, 1950.

Cervera, M. A., Thomas, R. M., Groves, K. M., Ramli, A. G., and Effendy: Validation of WBMOD in the Southeast Asian region, Radio Sci., 36, 1559-1571, 2001.

Fang, D. J. and Liu, C. H.: A morphological study of gigahertz equatorial scintillations in the Asian region, Radio Sci., 18, 241252, 1983.

Fejer, B. G., Scherliess, L., and dePaula, E. R.: Effects of the vertical plasma drift velocity on the generation and evolution of equatorial spread F, J. Geophys. Res., 104, 19859-19869, 1999.

Franke, S. J., Liu, C. H., and Fang, D. J.: Multifrequency study of ionospheric scintillation at Ascension Island, Radio Sci., 19, 695-706, 1984.

Fraser, D. J.: Scintillation Measurements from Ascension Island: Hardware \& Software Development and Deployment Report, Technical Report, QINETIQ/KI/COM/TN050963, QinetiQ, Malvern, UK, 2004.

Fraser, D., Cannon, P., Rogers, N., and Angling, M.: An initial study of kilometre-scale TEC irregularities in the context of space-based radar performance, Research Report, QINETIQ/05/01760, QinetiQ, Malvern, UK, 2005. 
Groves, K. M., Basu, S., Weber, E. J., Smitham, M., Kuenzler, H., Valladares, C. E., Sheehan, R., MacKenzie, E., Secan, J. A., Ning, P., McNeill, W. J., Moonan, D. W., and Kendra, M. J.: Equatorial scintillation and systems support, Radio Sci., 32, 2047-2064, 1997.

Livingston, R. C., Rino, C. L., McClure, J. P., and Hanson, W. B.: Spectral Characteristics of Medium-Scale Equatorial F Region Irregularities, J. Geophys. Res., 86, A4, 2421-2428, 1981.

Mullen, J. P., MacKenzie, E., Basu, S., and Whitney, H.: UHF/GHz scintillation observed at Ascension Island from 1980 through 1982, Radio Sci, 20, 357-365, 1985.

Nickisch, L. J.: A power law power spectral density model of total electron content structure in the polar region, Radio Sci., 39, RS1S12, doi:10.1029/2002RS002818, 2004.

Nishioka, M., Saito, A., and Tsugawa, T.: Occurrence characteristics of plasma bubble derived from global ground-based GPS receiver networks, J. Geophys. Res., 113, A05301, doi:10.1029/2007JA012605, 2008.

Rino, C. L.: A power law phase screen model for ionospheric scintillation, 1. Weak scatter, Radio Sci., 14, 1135-1145, 1979a.

Rino, C. L.: A power law phase screen model for ionospheric scintillation, 2 Strong scatter, Radio Sci., 14, 1147-1155, 1979b.

Rino, C. L., Tsunoda, R. T., Petriceks, J., Livingston, R. C., Kelley, M. C., and Baker, K. D.: Simultaneous Rocket-Borne Beacon and In Situ Measurements of Equatorial Spread F - Intermediate Wavelength Results, J. Geophys. Res., 86(A4), 2411-2420, 1981.
Rino, C. L.: On the application of phase screen models to the interpretation of ionospheric scintillation data, Radio Sci., 17(4), 855-867, 1982.

Rumsey, V. H.: Scintillations due to a concentrated layer with a power-law turbulence spectrum, Radio Sci., 10(1), 107-114, 1975.

Secan, J. A.: WBMOD ionospheric scintillation model - An abbreviated user's guide, NWRA-CR-94-R127/R2, NorthWest Research Associates, Bellevue, Washington, USA, 1996.

Van de Kamp, M. M. J. L., Cannon, P. S., and Terkildsen, M.: The effect of the Ionosphere on Defocusing of Space Based Radars, Radio Sci., 44, RS1003, doi:10.1029/2007RS003808, 2009.

Van Dierendonk, A. J., Klobuchar, J., and Hua, Q.: Ionospheric scintillation monitoring using commercial single frequency C/A code receivers, ION GPS-93, Proceedings: Sixth International Technical Meeting of the Satellite Division of the Institute of Navigation, Arlington, Virginia, USA, 1315-1322, 1993.

Wernik, A. W., Alfonsi, L., and Materassi, M.: Scintillation modeling using in situ data, Radio Sci., 42, RS1002, doi:10.1029/2006RS003512, 2007. 